\title{
The effect of the dust size distribution on asteroid polarization
}

\author{
Joseph Masiero $^{1}$, Christine Hartzell ${ }^{2}$, Daniel J. Scheeres ${ }^{2}$
}

\begin{abstract}
We have developed a theoretical description of how of an asteroid's polarizationphase curve will be affected by the removal of the dust from the surface due to a sizedependent phenomenon such as radiation pressure-driven escape of levitated particles. We test our calculations against new observations of four small ( $D \approx 1 \mathrm{~km})$ nearEarth asteroids [(85236), (142348), (162900) and $\left.2006 \mathrm{SZ}_{217}\right]$ obtained with the Dual Beam Imaging Polarimeter on the University of Hawaii's $2.2 \mathrm{~m}$ telescope, as well as previous observations of (25143) Itokawa and (433) Eros. We find that the polarization of the light reflected from an asteroid is controlled by the mineralogical and chemical composition of the surface and is independent of dust particle. The relation between the slope of the polarization-phase curve beyond the inversion angle and the albedo of an asteroid is thus independent of the surface regolith size distribution and is valid for both Main Belt and Near-Earth asteroids.
\end{abstract}

Subject headings: Minor planets, asteroids; Polarization

\section{Introduction}

The scattering of light can induce a polarization to an otherwise unpolarized photon flux. In the case of asteroids, the dust and regolith on the surface act to instill a linear polarization on the reflected sunlight. The degree of this polarization changes with phase angle, albedo, spectral type, and surface composition (Muinonen et al. 2002a). While single-particle scattering will always result in polarization perpendicular to the scattering plane ("positive" polarization), polarization in the scattering plane ("negative" polarization) is observed for very small phase angles for a wide range of objects (e.g. Dollfus \& Zellner 1979, etc.). Shkuratov et al. (1994) present a review of possible physical causes of this effect, the most promising of which are coherent backscattering models (e.g. Muinonen 1989) which also can describe the photometric opposition effect.

In this paper, we determine the effects of different size distributions of surface dust on the observed relationship between the polarization of the reflected light and the phase angle of the asteroid (that is, the Sun-asteroid-Earth angle). A variety of processes can cause dust loss on

\footnotetext{
${ }^{1}$ Institute for Astronomy, University of Hawaii, 2680 Woodlawn Dr, Honolulu, HI 96822, masiero@ifa.hawaii.edu

${ }^{2}$ Department of Aerospace Engineering Sciences, University of Colorado at Boulder, 429 UCB, Boulder, CO 80309-0429, christine.hartzell, scheeres@colorado.edu
} 
asteroids. Electrostatic charging of surface dust particles by Solar radiation, for example, can levitate smaller particles meters above the surface (Colwell et al. 2005, and reference therein). For small bodies these effects will result in the preferential loss of the smallest dust grains, effectively truncating the original dust size distribution. Here we test six cases of small near-Earth asteroids (NEAs), for which the dust loss should be more pronounced, and measure the effect of dust loss on the polarization-phase relation.

\section{Grain size limits}

The surfaces of most atmosphereless bodies in the inner Solar system are composed of a loose regolith ranging in size from dust to boulders. On the moon the smallest particles are dust down to $0.02 \mu \mathrm{m}$ in size (Tavlor et al. 2009) while for smaller asteroids such as (25143) Itokawa the regolith appears significantly coarser, with an apparent lack of anything smaller than $1 \mathrm{~mm}$ (Fujiwara et al. 2006). With a surface gravity significantly higher than all but the few largest asteroids, the dust distribution on the moon sets a strong lower-limit on the dust particle sizes we will consider in this study. As we will show in $\S$, the polarization-phase relation is insensitive to the upper-limit of the grain size distribution as long as that limit is $>10 \mu \mathrm{m}$.

Dust particles lofted from the surface of an asteroid by micro-meteoroid bombardment or electrostatic levitation are immediately subject to Solar radiation pressure effects. This pressure can put the particle into orbit around the parent body or allow the particle to escape into a heliocentric orbit. A lofted particle's motion can be described by Eq 1 which includes the effects of both gravity due to the asteroid (where $G$ is the universal gravitational constant, $M$ the mass of the asteroid, and $\mathbf{r}$ the position vector of the particle in $x, y, z$ space) and Solar radiation pressure $\left(g_{s}\right)$. The radiation pressure is assumed to act in the anti-Sun direction, $\hat{\mathbf{x}}$, with no time dependency (i.e. the $\mathrm{x}$-axis corresponds to the Sun-asteroid line and the orbit of the asteroid around the Sun is not included in this approximation). The Solar radiation pressure is described by Eq 2, where $p_{v}$ is the albedo of the particle (assumed to be zero initially), $C_{s}$ is a constant of Solar radiation $\left(1 \times 10^{7} \mathrm{~g} \mathrm{~km}^{3} /\left(\mathrm{s}^{2} \mathrm{~cm}^{2}\right)\right), B$ is the mass-to-area ratio of the particle, and $R$ is the distance to the Sun. The motion of the particle can then be described by the potential given in Eq 3 .

$$
\begin{aligned}
\ddot{\mathbf{r}} & =-G \frac{M}{r^{3}} \mathbf{r}+g_{s} \hat{\mathbf{x}} \\
g_{s} & =\frac{\left(1+p_{v}\right) C_{s}}{B R^{2}} \\
U & =G \frac{M}{r}+g_{s}(\hat{\mathbf{x}} \cdot \mathbf{r})
\end{aligned}
$$

A mathematical analysis of the orbital dynamics of test particles at an asteroid has been carried out previously by several authors. Dankowicz (1994) developed a precise limit on semi-major axis

of an orbiting particle under the assumption that the asteroid was stationary. Scheeres \& Marzari 
(2002) incorporated the motion of the asteroid about the Sun and developed a sufficient condition on semi-major axis for a particle (in this case a spacecraft) to be trapped in orbit about a small body. Byram \& Scheeres (2009) combined elements of these two earlier studies to derive more precise limits on semi-major axis of an orbiting test particle accounting for its motion. For purposes of this paper the analysis in Byram \& Scheeres (2009) is too detailed to be summarized simply, and thus we will only restate the results of these earlier papers.

From the analysis by Dankowicz (1994) we find that a simple limit exists for the semi-major axis of a lofted particle that generally ensures escape from the small body if exceeded. Mapped into our notation we find that escape will occur if the semi-major axis is larger than $a_{\text {escape }}$ :

$$
\begin{aligned}
a_{\text {escape }} & =\frac{\sqrt{3}}{4} \sqrt{G \frac{M}{g_{s}}} \\
& =\frac{\sqrt{3}}{4} \sqrt{G \frac{M B}{C_{s}}} R
\end{aligned}
$$

assuming an albedo of $p_{v}=0$. Using a very different approach, Scheeres \& Marzari (2002) developed a sufficient condition for stability about a small body and found that escape is not immediately possible if the semi-major axis is less than $a_{\min }$ :

$$
a_{\text {min }}=\frac{1}{4} \sqrt{G \frac{M B}{C_{s}}} R
$$

In the general problem it is possible for the semi-major axis to evolve, but we ignore this effect for this derivation. We note that these limiting semi-major axes scale linearly with the small body distance from the Sun. Thus, escape is most likely to occur at perihelion.

In order to calculate the mass-to-area ratio $(B)$ of a particle, we assume that it is spherical with a circular reflective surface area, giving $B=\frac{2}{3} \rho_{d} d_{d}$, where $\rho_{d}$ is the dust density and $d_{d}$ is the diameter of the dust particle. Note that these assumptions are conservative as spheres maximize the volume-to-area ratio, and thus these calculations provide a lower limit to the cross-sectional surface area of particles escaping. Additionally, we are ignoring any timescale for particle loss once in orbit, assuming that sufficient time has passed to clear all particles in the perihelion size-limit. From this we can expand Eq 2 to find:

$$
g_{s}=\frac{3}{2} \frac{\left(1+p_{v}\right) C_{s}}{\rho_{d} d_{d} R^{2}}
$$

Substituting Eq 7 into Eq 4 and assuming that the particle of interest rests on the asteroid's surface ( $2 a=D_{a s t} / 2$, where $D_{a s t}$ is the asteroid's diameter), we can develop an expression that relates the minimum particle size expected to be found on an asteroid's surface to the physical characteristics of the asteroid:

$$
d_{d} \leq \frac{3 C_{s}}{\pi G} \frac{p_{v}+1}{D_{a s t} \rho_{a s t} \rho_{d} R^{2}}
$$


Equation 8 shows that as the size of the central body decreases or as perihelion distance becomes smaller, the minimum surface particle size increases. This can be seen in Fig 1, which shows with thick lines the relationship given in Eq 8 (normalized by the central body diameter) assuming the central body and the dust particle have the same density and an albedo of $p_{v}=0.2$. We note that dust should have densities up to a factor of 2 more than the bulk asteroid density, however this is mitigated by the assumption of spherical shapes for particle size. For asteroids spinning near their disruption rate we can assume that $a=D_{a s t} / 2$, resulting in a minimum particle size that is a factor of four larger than predicted by Eq 8 .

Table 1 shows the particle size limit for (433) Eros and Itokawa, ignoring any rotation. Note that the minimum particle size is inversely related to the asteroid density, diameter and distance from the Sun. If we consider the migration of Itokawa from the Main Belt to its current location, we see that as the asteroid approaches the Sun, increasingly larger particles are able to escape. If the polarization of light reflected from an asteroid is dependent on the size of the particles on the surface we would expect NEAs to show changes in their polarization-phase curve as a function of the predicted particle size.

\section{Polarization Effects}

The coherent backscatter mechanism, a second order light-scattering effect, has been shown to be the dominant cause of both the observed polarization signature of light reflected from asteroids at small phase angles as well as the photometric opposition effect (Muinonen et al. 2002a). As phase angle increases this transitions to a regime dominated by first-order single-particle scattering. Using numerical techniques to calculate the scattering of individual surface elements, Muinonen et al. (2002b) find that the polarization of the light can be approximated as:

$$
P \sim \frac{\alpha^{2}}{2 n}-\left(\frac{n-1}{n+1}\right)^{2} \frac{(k d \alpha)^{2}}{2\left[1+(k d \alpha)^{2}\right]}
$$

where $P$ is the percent polarization, $\alpha$ is the phase angle in radians, $n$ is the index of refraction,

$k=\frac{2 \pi}{\lambda}$ is the wave number, and $d$ is the scatter separation distance. This assumes a single spacing for all scattering particles. Other recent models (e.g. Boehnhardt et al. 2004; Bagnulo et al. 2006) have assumed two discrete scattering components co-mixed at different weightings to fit observed polarization phase curves.

In this work we will instead consider a continuous distribution of grain spacings. The distribution of voids in a silicate glass follows an approximate power-law falloff for sizes beyond some critical peak void size (Malavasi, et al. 2006). We begin by assuming that the spacing between dust on an asteroid's surface also follows a power law, and that the voids have the same minimum and maximum characteristic sizes as the dust. This implies that chemical and mineralogical effects are fully incorporated into the size of the constituent grains, which we discuss further in $\S 6$.

Bottke et al. (2005) show that for their model NEA population the size distribution is fit by 
the power law $N \propto D^{-2.5}$ for sizes from $100 \mathrm{~m}$ to $<10 \mathrm{~cm}$. Using a large-area camera network, Halliday et al. (1996) find that the mass distribution of fireballs $(0.1 \leq M \leq 12 \mathrm{~kg})$ entering the Earth's atmosphere has an exponential power between -0.5 and -1 , translating to a size distribution power between -1.5 to -3 . We focus on this range of size distribution powers for our simulations.

By integrating Eq 9 over all grain sizes from $d_{\min }$ to $d_{\max }$ we obtain:

$$
P=A \int_{d_{\min }}^{d_{\max }} \frac{\alpha^{2}}{2 n}-\left(\frac{n-1}{n+1}\right)^{2} \frac{(k \bar{d} \alpha)^{2}}{2\left[1+(k \bar{d} \alpha)^{2}\right]} \bar{d}^{p} d \bar{d}
$$

where $A$ is a variable used to fit the depth of the negative polarization, $p$ is the exponential power of the grain-size distribution, and $d_{\min }$ and $d_{\max }$ are minimum and maximum sizes of the grain-size distribution in microns, respectively.

If we consider only a single wavelength (in this case $\lambda \approx 600 \mathrm{~nm}$, meaning $k=10 \mu \mathrm{m}^{-1}$ ) we find that the arbitrary variable $A$ reduces to a simple expression of $d_{m i n}$, giving:

$$
P=1.2 d_{\min } \int_{d_{\min }}^{d_{\max }} \frac{\alpha^{2}}{2 n}-\left(\frac{n-1}{n+1}\right)^{2} \frac{(10 \bar{d} \alpha)^{2}}{2\left[1+(10 \bar{d} \alpha)^{2}\right]} \bar{d}^{p} d \bar{d}
$$

Figure $2 \mathrm{a}$ shows the change in the polarization-phase relation for fixed $n, d_{\min }$ and $p$. As $d_{\text {max }}$ increases its effect on the curve diminishes, meaning that the polarization is insensitive to the largest particle size for general distributions. For all other simulations we fix the maximum particle size at $10 \mu \mathrm{m}$ to limit computation time. In Fig $2 \mathrm{~b}$ we plot the effects of a variable $d_{\min }$. As a normalizing agent, $d_{\min }$ has a strong effect on the slope of the relation beyond the inversion angle (the point at which the polarization-phase curve recovers to zero), but also on the shape and depth of the negative branch of polarization.

A variable index of refraction most significantly affects the location of the inversion angle $\alpha_{0}$. As shown in Fig $2 \mathrm{r}$ small changes in the index of refraction result in major changes to the inversion angle and depth of negative polarization, with only minor effects on the slope or shape of the negative branch. Finally, the effect of variations in the power of the size distribution are shown in Fig 2 d. For the ranges of powers considered there are only minor changes to the slope and inversion angle, and only in the extreme case is there a significant change to the negative polarization branch.

From Eq 11 we can calculate the properties of the polarization-phase relation in terms of physical constants only. We find that the slope for $\alpha>\alpha_{0}$ is described by:

$$
h=\frac{-1.275}{n(p+1)} d_{\min }\left(d_{\min }^{p+1}-d_{\max }^{p+1}\right)
$$

where $h$ is the slope (in percent polarization per degree). As $d_{\max }$ becomes large $(>>10 \mu \mathrm{m})$ it's effect on $h$ becomes negligible. 
Similarly, for $n \geq 1.5$ and $d_{\max } \geq 10 \mu \mathrm{m}$ we can describe the location of the inversion angle approximately as:

$$
\alpha_{0} \approx \sqrt{n\left(\frac{n-1}{n+1}\right)^{2}-\frac{1}{2\left(10 d_{\text {min }}\right)^{2}}}
$$

where $\alpha_{0}$ is the inversion angle in radians.

Cellino et al. (1999) derive updated constants for the relationship between polarization slope $h$ and geometric albedo $p_{v}$ :

$$
\log p_{v}=-1.118 \log h-1.779
$$

Using Eq 12 and assuming that $d_{\max }>>10 \mu \mathrm{m}$ we can now describe the geometric albedo of an asteroid as:

$$
\log p_{v}=1.118 \log \left(n(-p-1) d_{\text {min }}{ }^{-p-2}\right)-1.896
$$

\section{Observations}

In order to test the effect of dust depletion on the polarization of small NEAs, we conducted observations of four targets over a five week period at the end of 2008 using the Dual-Beam Imaging Polarimeter (DBIP) on the University of Hawaii's $2.2 \mathrm{~m}$ telescope (Masiero et al. 2007). DBIP is designed to measure both linear and circular polarizations of asteroids and other point sources at magnitudes $10 \leq V \leq 17$ with errors less than $0.1 \%$ (Masiero et al. 2008).

Targets were chosen from all small NEAs $(D \approx 1 \mathrm{~km})$ that would be brighter than $V=18$ over the observing window. Four asteroids had polarimetric measurements of sufficient quality to compare with the theory discussed above. Asteroids (85236), (142348), (162900) and $2006 \mathrm{SZ}_{217}$ were measured at a range of phase angles sufficient to determine both $\alpha_{0}$ and $h$. Table 2 gives the target name, UT date of observation, apparent $V$ magnitude, exposure time, number of exposures, Solar phase angle $\alpha$, measured linear polarization, and angle of polarization referenced to the vector orthogonal to the Sun-object-Earth scattering plane.

In Table 3 we present for each asteroid the fitted inversion angle and slope (considering only phase angles $\alpha \geq 15^{\circ}$ ) as well as perihelion distance (q), absolute magnitude $\left(\mathrm{H}_{v}\right.$ ), albedo (as calculated from Eq 14), diameter (D), $n$ and $d_{\min }$ calculated from Eqs 12 and 13 (assuming $p=-3$ and $\left.d_{\max }=10\right)$, and the range of predicted minimum particle sizes remaining on the surface $\left(d_{\text {pred }}\right)$ depending on the assumed density (between 1.5 and $3.0 \mathrm{~g} \mathrm{~cm}^{-3}$ ) following $\S$. Included in the table are the same values for Eros (Zellner \& Gradie 1976) and Itokawa (Cellino et al. 2005), two NEAs that have been extensively studied both remotely as well as in situ by spacecraft visits. A measured density for each of these objects (Cheng 2002; Abe et al. 2004) allows us to make a singular prediction for minimum surface particle size. The $d_{\text {pred }}$ range or value for each of the six 
asteroids is shown in Fig 1, Scaling the levitation calculations from Colwell et al. (2005) to smaller asteroids we find our predicted minimum particle sizes are within the range of particle sizes that are capable of being levitated, assuming the particle's surface electric potential is equal to that of the asteroid's surface.

Figure 3 shows our observations and fits for each asteroid, as well as for the literature data for Itokawa and Eros. Note that the fits for $2006 \mathrm{SZ}_{217}$ and (85236) are under-constrained, thus values for the slope have large errors and the inversion angles are only approximate. In all cases, the polarization-phase relation beyond $\alpha_{0}$ was assumed to be strictly linear.

\section{Results and Discussion}

Calculations of the dust retention for the NEAs investigated predict over two orders of magnitude in variation of the minimum particle size. We find no correlation in our data between the $d_{\text {min }}$ calculated from our measured slope and the predicted minimum particle size. If the individual dust particles were unique scattering elements we would expect the opposite to be true. Thus the physical size of the dust cannot be the primary cause of polarization or even a significant contributor.

In fact, (85236), (162900), $2006 \mathrm{SZ}_{217}$, Itokawa and Eros all show slopes and inversion angles consistent with those measured for large S-type Main Belt asteroids (MBAs), while (142348) shows polarization similar to what is seen for large C-type MBAs (see, e.g Muinonen et al. 2002a). From this we infer that the polarization of light reflected from an asteroid is constrained primarily by the way the local mineralogy and chemistry dictate the formation of spaces in the mineral matrix. The calculated value of $d_{\min }$ then represents not the size of the smallest particle on the surface, but instead a characteristic minimum size of mineralogical voids. For asteroids with similar spectral signatures, and thus similar surface chemistries, we expect to see correspondingly similar polarization properties. Asteroid polarization-phase curves should show no variation with changing regolith conditions for asteroids of the same composition.

The polarization-albedo relation (Eq 14) then does not depend on surface regolith size distribution and so is identical for NEAs and MBAs. Variations in surface regolith and observing geometries between NEAs and MBAs require thermal models tailored for each population (Harris 1998; Wolters \& Green 2009) which in turn affects infrared albedo determinations. Polarimetrically determined albedos are therefore immune to this complication and can be robustly applied to any asteroid regardless of location in the Solar system. 


\section{Conclusions}

Through modeling of the effects of a distribution of spacings between scattering elements on the observed polarization-phase relation, we have shown that the relation is independent of the size of the particles on the surface. Instead, the slope of the relation beyond the inversion angle (and thus the spacing parameter $d$ ) remains constant for a range of objects with dust-retention sizes spanning two orders of magnitude. The polarization-phase relation is therefore consistent between MBAs and NEAs of the same spectral type. An asteroid's surface chemistry and mineralogy are the dominant cause of the polarization-phase relation, and this relation is equally applicable for both NEAs and MBAs.

\section{Acknowledgments}

We would like to thank Robert Jedicke and the anonymous referee for their comments on the paper that greatly improved the text. J.M. was supported under NASA PAST grant NNG06GI46G. D.J.S. acknowledges support from NASA's Discovery Data Analysis Program. The authors wish to recognize and acknowledge the very significant cultural role and reverence that the summit on Mauna Kea has always had within the indigenous Hawaiian community. We are most fortunate to have the opportunity to conduct observations from this sacred mountain.

\section{REFERENCES}

Abe, S., Mukai, T., Hirata, N., et al., 2006, "Mass and Local Topography Measurements of Itokawa by Hayabusa", Science, 312, 1344.

Bagnulo, S., Boehnhardt, H., Muinonen, K., Kolokolova, L., Belskaya, I. \& Barucci, M. A., 2006, "Exploring the surface properties of transneptunian objects and Centaurs with polarimetric FORS1/VLT observations", A\&A, 450, 1239.

Boehnhardt, H., Bagnulo, S., Muinonen, K., Barucci, M. A., Kolokolova, L., Dotto, E. \& Tozzi, G. P., 2004, "Surface characterization of 28978 Ixion $\left(2001 \mathrm{KX}_{76}\right)$ ", A\&A, 415L, 21.

Bottke, W.F., Durda, D.D., Nesvorný, D., Jedicke, R., Morbidelli, A., Vokrouhlický, D. \& Levison, H.F., 2005, "Linking the collisional history of the main asteroid belt to its dynamical excitation and depletion", Icarus, 179, 63.

Byram, S.M. \& Scheeres, D.J., 2009, "Stability of Sun-Synchronous Orbits in the Vicinity of a Comet", Journal of Guidance, Control and Dynamics, in press.

Cellino, A., Yoshida, F., Anderlucci, E., et al., 2005, "A polarimetric study of Asteroid 25143 Itokawa", Icarus, 179, 297. 
Cellino, A., Gil Hutton, R., Tedesco, E.F., di Martino, M. \& Brunini, A., 1999, "Polarimetric Observations of Small Asteroids: Preliminary Results", Icarus, 138, 129.

Cheng, A.F., 2002, "Near Earth Asteroid Rendezvous: Mission Summary", Asteroids III, ed. Bottke, Cellino, Paolicchi \& Binzel (Univ of Arizona Press), 351.

Colwell, J.E., Gulbis, A.A.S., Horányi, M. \& Robertson, S., 2005, "Dust transport in photoelectric layers and the formation of dust ponds on Eros", Icarus, 175, 159.

Dankowicz, H., 1994, "Some special orbits in the two-body problem with radiation pressure", Celestial Mechanics and Dynamical Astronomy, 58, 353.

Dollfus, A. \& Zellner, B., 1979, "Optical polarimetry of asteroids and laboratory samples", Asteroids (Univ of Arizona Press), 170.

Fujiwara, A., Kawaguchi, J., Yeomans, D.K., et al., 2006, "The Rubble-Pile Asteroid Itokawa as Observed by Hayabusa", Science, 312, 1330.

Halliday, I., Griffin, A.A. \& Blackwell, A.T., 1996, "Detailed data for 259 fireballs from the Canadian camera network and inferences concerning the influx of large meteoroids", M\&PS, 31, 185.

Harris, A.W., 1998, "A Thermal Model for Near-Earth Asteroids", Icarus, 131, 291.

Malavasi, G., Menziani, M.C., Pedone, A. \& Segre, U., 2006, "Void size distribution in MD-modelled silica glass structures", Journal of Non-Crystalline Solids, 352, 285.

Masiero, J., Hodapp, K.-W., Harrington, D. \& Lin, H., 2007, "Commissioning of the Dual-Beam Imaging Polarimeter for the UH 88-inch telescope", PASP, 119, 1126.

Masiero, J., Hodapp, K.-W., Harrington, D. \& Lin, H., 2008, "Extended Commissioning and Calibration of the Dual-Beam Imaging Polarimeter", to appear in ASP Conf. Series for Astronomical Polarimetry 2008; arXiv:0809.4313.

Muinonen, K., 1989, "Electromagnetic Scattering by Two Interacting Dipoles", Proc. URSI International Symp. on Electromagnetic Theory, 428.

Muinonen, K., Piironen, J., Shkuratov, Y., Ovcharenko, A. \& Clark, B., 2002a, "Asteroid Photometric and Polarimetric Phase Effects", Asteroids III, ed. Bottke, Cellino, Paolicchi \& Binzel (Univ of Arizona Press), 123.

Muinonen, K., Videen, G., Zubko, E. \& Shkuratov, Yu., 2002b, "Numerical Techniques for Backscattering by Random Media", Optics of Cosmic Dust, ed. Videen \& Kocifaj (NATO Science Series), 79, 261.

Scheeres, D.J. \& Marzari, F., 2002, "Spacecraft Dynamics in the Vicinity of a Comet", Journal of the Astronautical Sciences, 50, 35. 
Shkuratov, Yu.G., Muinonen, K., Bowell, E., Lumme, K., Peltoniemi, J.I., Kreslavsky, M.A., Stankevich, D.G., Tishkovetz, V.P., Opanasenko, N.V., \& Melkumova, L.Y., 1994, "A Critical Review of Theoretical Models of Negatively Polarized Light Scattered by Atmosphereless Solar System Bodies", EM\&P, 65, 201.

Taylor, L.A., Liu, Y. \& Zhang, A., 2009, "Shape and Size Relationship of Several Lunar Dusts: Preliminary Results", LPI, 40, 2106.

Wolters, S.D. \& Green, S.F., 2009 "Investigation of Systematic Bias in Radiometric Diameter Determination of Near-Earth Asteroids: the Night Emission Simulated Thermal Model (NESTM)", arXiv:0905.1601.

Zellner, B. \& Gradie, J., 1976 "Polarization of the reflected light of asteroid 433 Eros", Icarus, 28, 117. 
Table 1. Calculated minimum particle sizes from the dynamical model

\begin{tabular}{lcccc}
\hline \hline & $\begin{array}{c}\text { Eros } \\
\text { Perihelion }\end{array}$ & $\begin{array}{c}\text { Itokawa } \\
\text { Main Belt }\end{array}$ & $\begin{array}{c}\text { Itokawa } \\
\text { Aphelion }\end{array}$ & $\begin{array}{c}\text { Itokawa } \\
\text { Perihelion }\end{array}$ \\
\hline Asteroid Diameter $(\mathrm{km})$ & 16.84 & 0.33 & 0.33 & 0.33 \\
Distance from Sun $(\mathrm{AU})$ & 1.133 & $2.500^{a}$ & 1.695 & 0.953 \\
Density $\left(\mathrm{g} / \mathrm{cm}^{3}\right)$ & 2.7 & 1.9 & 1.9 & 1.9 \\
Minimum Particle Size $(\mu \mathrm{m})$ & 0.5 & 10.3 & 22.4 & 71.0 \\
\hline \hline
\end{tabular}

Diameters and orbital distances from JPL/Horizons; density values from Cheng (2002); Abe et al. (2004); ${ }^{a}$ assumed value

Table 2. Asteroid Observations

\begin{tabular}{cccccccc}
\hline \hline \multirow{2}{*}{ Asteroid } & UT Obs Date & V mag & $\mathrm{T}_{\text {exp }}(\mathrm{sec})$ & $\mathrm{n}_{\text {exp }}$ & $\alpha$ & ${\text { Linear \% } \mathrm{Pol}^{a}}^{*} \theta_{p}$ \\
\hline \multirow{2}{*}{$2006 \mathrm{SZ}_{217}$} & $2008-11-18$ & 15.6 & 300 & 18 & $19.8^{\circ}$ & $0.1 \pm 0.2$ & $9 \pm 10$ \\
& $2008-12-3$ & 16.0 & 240 & 12 & $21.7^{\circ}$ & $0.3 \pm 0.1$ & $11 \pm 7$ \\
& $2008-12-23$ & 17.4 & 300 & 12 & $36.9^{\circ}$ & $1.4 \pm 0.5$ & $176 \pm 15$ \\
\multirow{3}{*}{62900} & $2008-11-18$ & 14.9 & 300 & 12 & $5.3^{\circ}$ & $-0.5 \pm 0.1$ & $86 \pm 13$ \\
& $2008-12-3$ & 14.9 & 120 & 12 & $16.4^{\circ}$ & $-0.2 \pm 0.1$ & $90 \pm 9$ \\
& $2008-12-23$ & 15.1 & 100 & 18 & $30.7^{\circ}$ & $1.1 \pm 0.1$ & $177 \pm 7$ \\
142348 & $2008-11-18$ & 16.2 & 300 & 6 & $39.3^{\circ}$ & $5.1 \pm 0.5$ & $177 \pm 4$ \\
& $2008-12-3$ & 16.1 & 270 & 18 & $32.6^{\circ}$ & $2.4 \pm 0.1$ & $2 \pm 2$ \\
& $2008-12-23$ & 16.1 & 270 & 6 & $17.1^{\circ}$ & $0.1 \pm 0.1$ & $20 \pm 11$ \\
85236 & $2008-12-3$ & 16.3 & 300 & 18 & $60.3^{\circ}$ & $3.8 \pm 0.1$ & $179 \pm 1$ \\
& $2008-12-23$ & 17.7 & 300 & 12 & $46.6^{\circ}$ & $2.5 \pm 0.5$ & $177 \pm 9$ \\
\hline \hline
\end{tabular}

${ }^{a}$ quoted errors are $1 \sigma$ statistical errors; systematic errors are $\approx 0.05 \%$. 
Table 3. Derived Physical and Dust Properties

\begin{tabular}{|c|c|c|c|c|c|c|c|c|c|}
\hline Asteroid & $\alpha_{0}(\operatorname{deg})$ & $\mathrm{h}$ & $\mathrm{q}(\mathrm{AU})$ & $\mathrm{H}_{V}$ & $p_{v}$ & $\mathrm{D}(\mathrm{km})$ & $n$ & $d_{\min }(\mu \mathrm{m})$ & $d_{\text {pred }}(\mu \mathrm{m})$ \\
\hline $2006 \mathrm{SZ}_{217}$ & $\sim 18$ & $0.08 \pm 0.03$ & 1.200 & 17.4 & $0.3_{-0.1}^{+0.2}$ & $0.8 \pm 0.2$ & $\sim 1.64$ & $5_{-2}^{+6}$ & $8-32$ \\
\hline 162900 & $19 \pm 3$ & $0.09 \pm 0.01$ & 1.215 & 15.8 & $0.24 \pm 0.03$ & $1.9 \pm 0.1$ & $1.7 \pm 0.1$ & $4.2_{-0.7}^{+0.9}$ & $3-13$ \\
\hline 142348 & $17 \pm 2$ & $0.15 \pm 0.01$ & 1.119 & 18.2 & $0.14 \pm 0.01$ & $0.81_{-0.02}^{+0.03}$ & $1.60 \pm 0.06$ & $2.6 \pm 0.2$ & $8-32$ \\
\hline 85236 & $\sim 18$ & $0.09 \pm 0.04$ & 0.850 & 18.5 & $0.2_{-0.1}^{+0.2}$ & $0.6 \pm 0.2$ & $\sim 1.65$ & $4_{-2}^{+5}$ & $20-79$ \\
\hline Itokawa & $20 \pm 0.2^{a}$ & $0.084 \pm 0.001^{a}$ & 0.953 & 19.2 & $0.265 \pm 0.002$ & $0.373 \pm 0.001^{c}$ & $1.72 \pm 0.01$ & $4.41_{-0.05}^{+0.03}$ & 62.8 \\
\hline Eros & $21.7 \pm 0.9^{b}$ & $0.107 \pm 0.002^{b}$ & 1.133 & 11.2 & $0.203 \pm 0.004$ & $17.0_{-0.2}^{+0.1 c}$ & $1.79 \pm 0.03$ & $3.3 \pm 0.1$ & 0.5 \\
\hline
\end{tabular}

${ }^{a}$ data from Cellino et al. (2005). ${ }^{b}$ data from Zellner \& Gradie (1976). ${ }^{c}$ calculated from polarimetry, thus differing slightly from in situ values. 


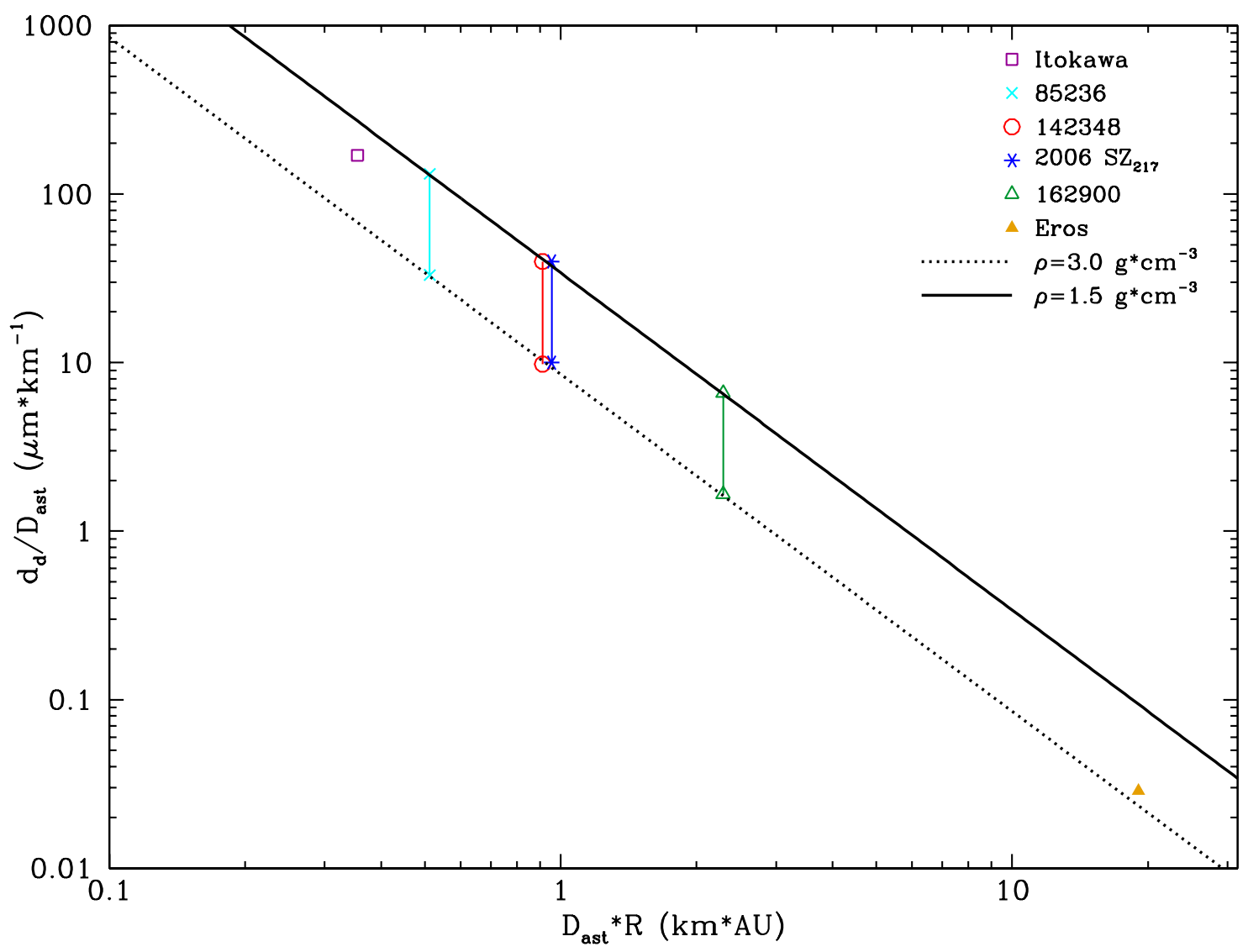

Fig. 1. - Ratio of the minimum dust particle size on the surface to the diameter of the asteroid, as related to the asteroid's diameter times the distance to the Sun. The solid and dotted lines show the relationship for constant densities of 1.5 and $3.0 \mathrm{~g} \mathrm{~cm}^{-3}$, respectively, when assuming an albedo of 0.2. The points indicate the positions of NEAs considered in this study with known densities, while the ranges represent NEAs modeled with densities from 1.5 to $3.0 \mathrm{~g} \mathrm{~cm}^{-3}$. Deviations of the calculated points from the lines of constant density are due to slight differences in the measured albedos. 

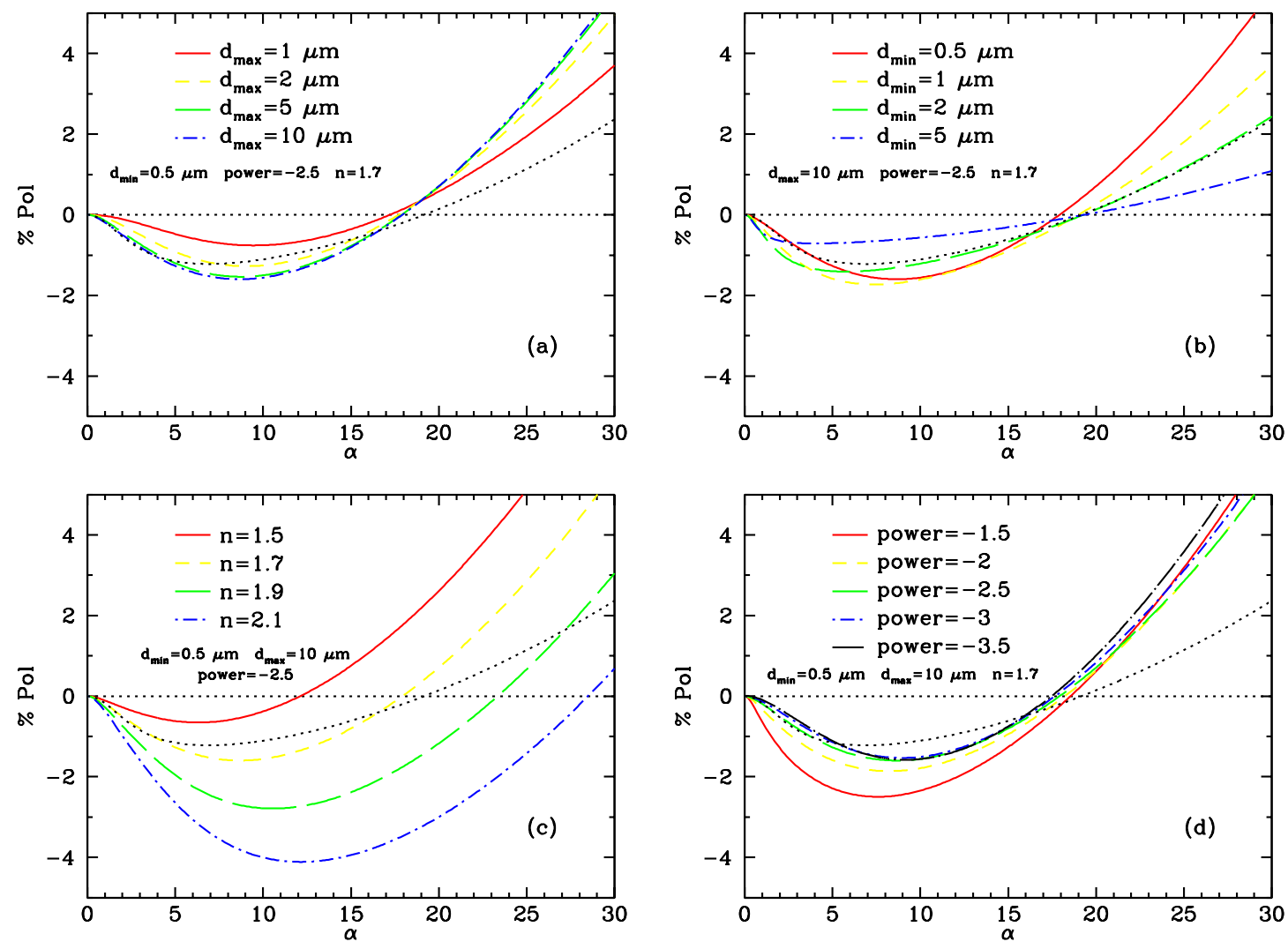

Fig. 2.- Simulated polarization-phase curves for variations in the value of (a) the maximum grain size, (b) the minimum grain size, (c) the index of refraction, and (d) the power of the size distribution. The curves show the relation between percent of incident light polarized, referenced to the normal to the scattering plane, and the Solar phase angle $(\alpha)$ measured in degrees. The dotted curve in each box shows the polarization described by Eq 9 for $n=1.7,(k d)=20$, and a normalizing constant of 50: a good approximation for the polarization from a typical S-type Main Belt asteroid. 

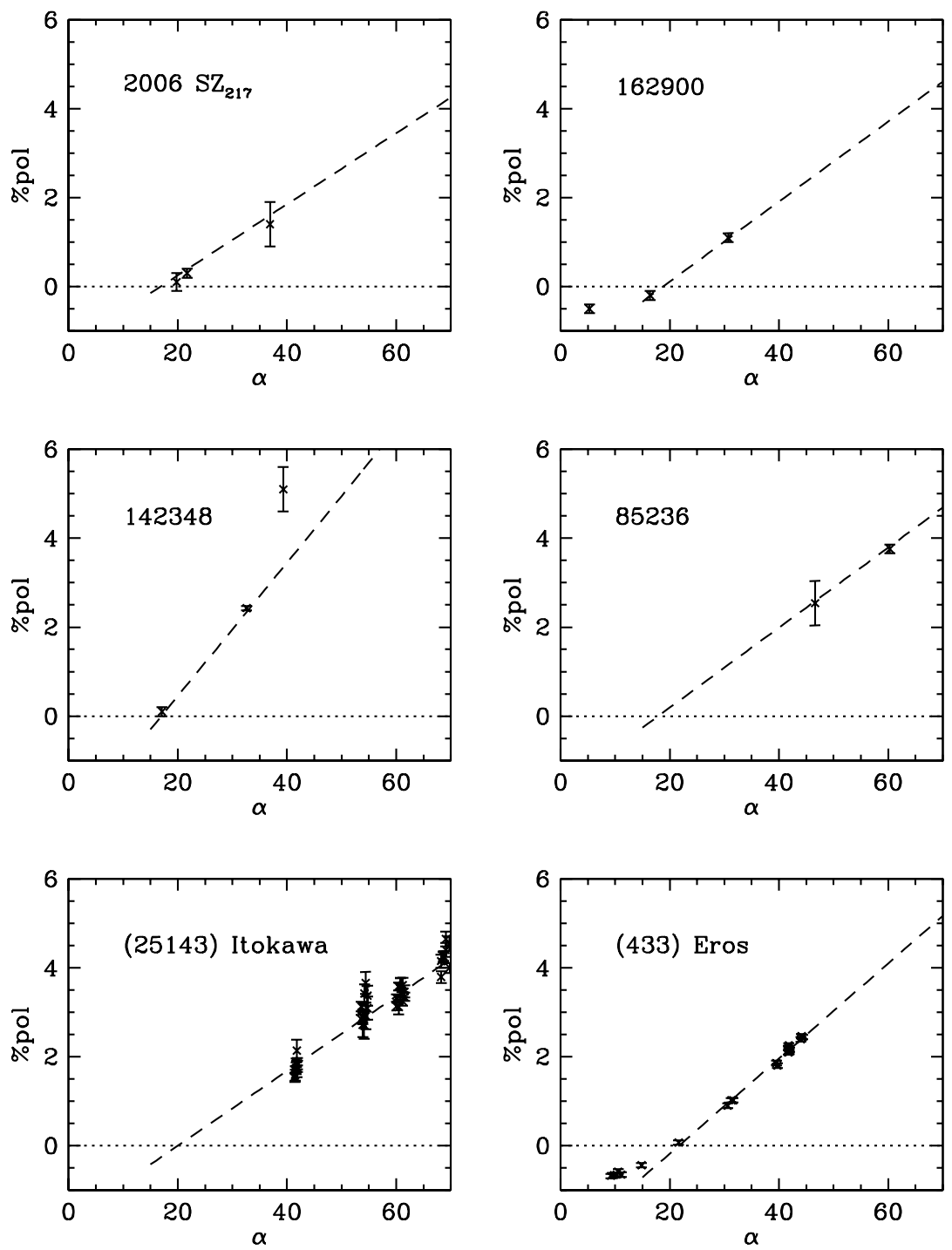

Fig. 3.- Observations from Table 2, as well as the best fitting linear polarization-phase relation for phase angles $\alpha \geq 15^{\circ}$ (dashed line). Fitted slopes and inversion angles are given in Table 3 . Note that $2006 \mathrm{SZ}_{217}$ and (85236) are under-constrained. Data for Itokawa is from Cellino et al. (2005) while data for Eros is from Zellner \& Gradie (1976). 